\title{
Ultrasound and Cartilage Thickness in Juvenile Idiopathic Arthritis: New Findings, New Questions
}
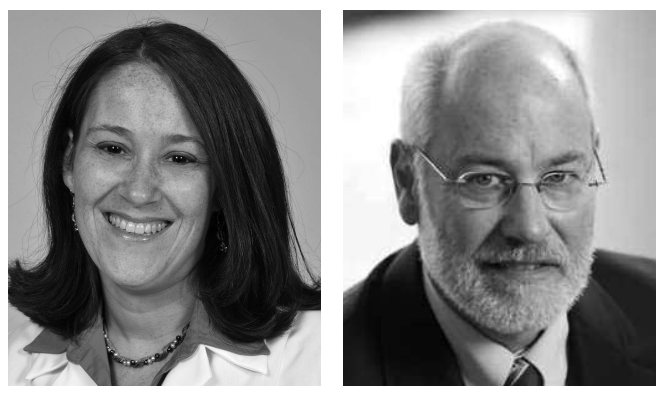

In recent years, ultrasound (US) has emerged as a potentially powerful tool for diagnosis and assessment in juvenile idiopathic arthritis (JIA). US with power Doppler (PD) can identify joint effusions, synovial hypertrophy, and increased vascularity, all of which suggest active synovitis. Chronic disease is demonstrated by structural joint damage, including erosions and cartilage thinning. These findings have been considered important markers for disease progression, and they can influence management. Current treatment paradigms aim to avoid reaching the point of structural damage; thus much of the focus of US in JIA has been on identifying active inflammation and subclinical disease $^{1}$. Studies have suggested that US may be more sensitive for identifying active disease than physical examination ${ }^{1,2}$.

The role of US in detecting structural damage in children, specifically cartilage loss, is complicated by the normal ossification process in the developing skeleton. Interpreting cartilage measurements requires an understanding of the variations in cartilage thickness based on age and sex. Spannow, et al have been at the forefront of establishing age- and sex-specific normal values for cartilage thickness over the past several years. Their initial study in 2009 assessed intrarater and interrater reliability when measuring cartilage thickness in children by US ${ }^{3}$. In 2010, they looked at a large cohort of 394 normal children and developed sex- and age-related normal values for cartilage thickness ${ }^{4}$. The finding that normal values varied by sex and age was also validated by our group using a slightly different technique ${ }^{5}$. Spannow, et al further validated their methods by comparing cartilage thickness measured on magnetic resonance imaging (MRI) and US in 25 healthy children, and found a good level of agreement between the 2 imaging methods ${ }^{6}$.

In this issue of The Journal, Pradsgaard, et al applied their techniques to JIA ${ }^{7}$. The stated goal of the authors was to assess for variations in cartilage thickness of specific joints in children with JIA based on category, disease duration, and disease activity, and to compare these results to normal children. Children between ages 5 and 15 years were examined, and US was performed in 10 preselected joints. Measurements were performed as described in the authors' previous studies. Ninety-five subjects with systemic JIA, oligoarticular JIA (persistent and extended), and polyarticular JIA (rheumatoid factor-positive or negative) were included and compared to age- and sex-related healthy children.

The authors found that after controlling for age and gender, cartilage thickness measured in the total JIA cohort was significantly lower in the knee, wrist, and second proximal interphalangeal joints (PIP) when compared with healthy controls. Further, cartilage thickness in the knee was significantly decreased in patients with polyarticular and systemic JIA compared to the oligoarticular subgroup. They did not analyze persistent versus extended oligo-JIA separately. Surprisingly, there was no significant difference in cartilage thickness between affected and unaffected joints on the same patient. Additionally, unaffected knees, wrists, and second PIP [but not ankles or second metacarpophalangeal (MCP) joints] also showed decreased thickness compared to healthy controls. Multiple intraarticular corticosteroid injections in the knee, ankle, and wrist did not appear to affect the mean cartilage thickness, supporting a previous study ${ }^{8}$.

It is not clear what accounts for the decreased cartilage thickness in unaffected joints. It would be useful to repeat this analysis by specific JIA category. While it seems plausible that children with systemic JIA and poly-JIA could have subclinical disease in unaffected joints causing this discrepancy, it is somewhat alarming to think that this could be occurring in patients with oligo-JIA. If this is the case, even in patients with oligo-JIA, it raises the question of whether we should change our approach to oligo-JIA patients by starting systemic treatment earlier. Consensus

See US detects decreased cartilage thickness in JIA, page 1596

Personal non-commercial use only. The Journal of Rheumatology Copyright (c) 2013. All rights reserved. 
guidelines published in 2011 endorsed by the American College of Rheumatology considered the number of active joints to be a pivotal factor in deciding whether or not to start systemic medication ${ }^{9}$. Historically, active joints have been defined as those with (1) non-bony swelling or (2) limitation with either pain on motion or tenderness. US is changing this definition, but the clinical implications remain somewhat unclear. Findings by Spannow, et al emphasize the need for continued clarification in this area.

The issue of whether or not US can detect subclinical disease is controversial. A recent study by Magni-Manzoni, et al looked at this issue with regard to US-detected synovial abnormalities in children with clinically inactive JIA. They examined 52 joints in clinically inactive patients for synovial hyperplasia, joint effusion, PD signal, and tenosynovitis and followed the children for 2 years to determine if joints deemed active on US (while quiet clinically) became active over time ${ }^{10}$. They found that the presence of synovial hyperplasia, joint effusion, PD signal, and tenosynovitis in a child in remission did not predict clinical flare. In that study, the majority of the patients had oligo-JIA (18 with persistent oligo-JIA, 12 with extended). Given these findings and the findings of Spannow's group, it is clear that more longitudinal studies are needed to better delineate which changes on US have meaningful clinical implications and thus require intervention. This is particularly important at a time when there is emphasis on early, aggressive treatment; knowing the clinical implications of our imaging findings could not only help us treat early but also help avoid overtreating.

Another surprising finding was that the group with disease duration $<1$ year had significantly decreased cartilage thickness compared to those with disease duration $>1$ year. This was found in the knee, ankle, and second PIP, but not in the wrist and second MCP. Joint space narrowing is considered an early indicator of damage ${ }^{11}$, and cartilage thinning is often thought to contribute to this finding. The data of Spannow, et al could suggest that some cartilage thinning is reversible. Alternatively, the acute disease process in active joints skews the appearance of cartilage such that it appears thinner early in the disease course but normalizes later on.

Advances in MRI techniques such as T1 $\rho$ and T2 mapping allow for quantitative assessment of cartilage damage and repair and do not require contrast injection. While traditional MRI techniques are useful for detecting morphological changes, the new modalities allow determination of proteoglycan content (T1 $\rho)$ and biochemical changes in cartilage (T2 mapping) ${ }^{12}$. Use of these techniques in the study of children with JIA may shed light on the observed relationships between cartilage thickness in affected and unaffected joints as well as in patients with shorter and longer durations of disease.

Correlation of MRI findings with US findings may offer new strategies for assessing disease activity and damage in the clinic and could have a substantial influence on treatment paradigms. Unfortunately, the data may be somewhat slow in coming because of the relative rarity of pediatric disease, the user-dependent component of US technique, and the cost. Ultimately, the goal is to improve patient care, add to our understanding of disease processes, increase efficiency, and decrease cost. The findings of Spannow, et al that do not fit neatly into our existing paradigm of cartilage damage in JIA and our understanding of the disease process will stimulate further investigation in this quickly changing area.

\section{GINGER JANOW, MD, MPH, Department of Pediatrics, Division of Pediatric Rheumatology, The Joseph M. Sanzari Children's Hospital, Hackensack, New Jersey; \\ NORMAN T. ILOWITE, MD, \\ Department of Pediatrics, Division of Pediatric Rheumatology, Children's Hospital at Montefiore, Bronx, New York, USA Address correspondence to Dr. Janow; E-mail: gjanow@hackensackumc.org}

\section{REFERENCES}

1. Janow GL, Panghaal V, Trinh A, Badger D, Levin TL, Ilowite NT. Detection of active disease in juvenile idiopathic arthritis: Sensitivity and specificity of the physical examination vs ultrasound. J Rheumatol 2011;38:2671-4.

2. Wakefield RJ, Green MJ, Marzo-Ortega H, Conaghan PG, Gibbon WW, McGonagle D, et al. Should oligoarthritis be reclassified? Ultrasound reveals a high prevalence of subclinical disease. Ann Rheum Dis 2004;63:382-5.

3. Spannow AH, Pfeiffer-Jensen M, Andersen NT, Stenbog E, Herlin T. Inter- and intraobserver variation of ultrasonographic cartilage thickness assessments in small and large joints in healthy children. Pediatr Rheumatol Online 2009;7:12.

4. Spannow AH, Pfeiffer-Jensen M, Andersen NT, Herlin T, Stenbog E. Ultrasonographic measurements of joint cartilage thickness in healthy children: Age- and sex-related standard reference values. J Rheumatol 2010;37:2595-601.

5. Panghaal V, Janow G, Trinh A, Ilowite N, Levin TL. Normal epiphyseal cartilage measurements in the knee in children: An alternative sonographic approach. J Ultrasound Med 2012;31:49-53.

6. Spannow AH, Stenboeg E, Pfeiffer-Jensen M, Fiirgaard B, Haislund M, Ostergaard M, et al. Ultrasound and MRI measurements of joint cartilage in healthy children: A validation study. Ultraschall Med 2011;32 Suppl 1:S110-6.

7. Pradsgaard DØ, Spannow AH, Heuck C, Herlin T. Decreased cartilage thickness in juvenile idiopathic arthritis assessed by ultrasonography. J Rheumatol 2013;40:1596.

8. Huppertz HI, Tschammler A, Horwitz AE, Schwab KO. Intraarticular corticosteroids for chronic arthritis in children: efficacy and effects on cartilage and growth. J Pediatr 1995; 127:317-21.

9. Beukelman T, Patkar NM, Saag KG, Tolleson-Rinehart S, Cron RQ, DeWitt EM, et al. 2011 American College of Rheumatology recommendations for the treatment of juvenile idiopathic arthritis: Initiation and safety monitoring of therapeutic agents for the

Personal non-commercial use only. The Journal of Rheumatology Copyright @ 2013. All rights reserved. 
treatment of arthritis and systemic features. Arthritis Care Res 2011;63:465-82.

10. Magni-Manzoni S, Scire CA, Ravelli A, Klersy C, Rossi S, Muratore V, et al. Ultrasound-detected synovial abnormalities are frequent in clinically inactive juvenile idiopathic arthritis, but do not predict a flare of synovitis. Ann Rheum Dis 2013;72:223-8.

11. Breton S, Jousse-Joulin S, Finel E, Marhadour T, Colin D, de
Parscau L, et al. Imaging approaches for evaluating peripheral joint abnormalities in juvenile idiopathic arthritis. Semin Arthritis Rheum 2012;41:698-711.

12. Choi JA, Gold GE. MR imaging of articular cartilage physiology. Magn Reson Imaging Clin N Am 2011;19:249-82.

J Rheumatol 2013;40:1466-8; doi:10.3899/jrheum.130737 